\title{
CONF-9507/30--1
}

Janis A. Keeney ${ }^{1}$ and John W. Bryson ${ }^{1}$

\section{STRESS-INTENSITY-FACTOR INFLUENCE COEFFICIENTS FOR SEMIELLIPTICAL INNER-SURFACE FLAWS IN CLAD PRESSURE VESSELS}

\begin{abstract}
REFERENCE: Keeney, J. A. and Bryson, J. W., "Stress-Intensity-Factor Influence Coefficients for Semielliptical Inner-Surface Flaws in Clad Pressure Vessels," Fracture Mechanics: 26th Volume, ASTM STP 1256, Walter G. Reuter, John H. Underwood, and James C. Newman, Jr., Eds., American Society for Testing and Materials, Philadelphia, 1995.
\end{abstract}

\begin{abstract}
A problem of particular interest in pressure vessel technology is the calculation of accurate stress-intensity factors for semielliptical surface cracks in cylinders. Computing costs for direct solution techniques can be prohibitive when applied to threedimensional (3-D) geometries with time-varying boundary conditions such as those associated with pressurized thermal shock. An alternative superposition technique requires the calculation of a set of influence coefficients for a given 3-D crack model that can be superimposed to obtain mode-I stress-intensity factors. This paper presents stressintensity-factor influence coefficients (SIFICs) for axially and circumferentially oriented finite-length semielliptical inner-surface flaws with aspect ratios [total crack length $(2 \mathrm{c})$ to crack depth (a)] of 2, 6, and 10 for clad cylinders having an internal radius $\left(R_{i}\right)$ to wall thickness $(\mathrm{t})$ ratio of 10 . SIFICs are computed for flaw depths in the range of $0.01 \leq \mathrm{a} / \mathrm{t} \leq$ 0.5 and two cladding thicknesses. The incorporation of this SIFIC data base in fracture mechanics codes will facilitate the generation of fracture mechanics solutions for a wide range of flaw geometries as may be required in structural integrity assessments of pressurized-water and boiling-water reactors.
\end{abstract}

KEYWORDS: stress-intensity-factor influence coefficient, pressurized thermal shock, finite-length flaw, reactor pressure vessel, superposition

Several techniques have been employed by analysts to calculate accurate stressintensity factors for semielliptical surface cracks in cylinders. Solution techniques that have been applied to this problem include direct three-dimensional (3-D) calculations using a

1Engineers, Oak Ridge National Laboratory, P. O. Box 2009, Oak Ridge, TN 378318056 . 


\section{DISCLAIMER}

This report was prepared as an account of work sponsored by an agency of the United States Government. Neither the United States Government nor any agency thereof, nor any of their employees, makes any warranty, express or implied, or assumes any legal liability or responsibility for the accuracy, completeness, or usefulness of any information, apparatus, product, or process disclosed, or represents that its use would not infringe privately owned rights. Reference herein to any specific commercial product, process, or service by trade name, trademark, manufacturer, or otherwise does not necessarily constitute or imply its endorsement, recommendation, or favoring by the United States Government or any agency thereof. The views and opinions of authors expressed herein do not necessarily state or reflect those of the United States Government or any agency thereof. 


\section{DISCLAIMER}

Portions of this document may be illegible in electronic image products. Images are produced from the best available original document. 
finite-element method [1,2]. However, computing costs for this direct approach can be prohibitive when applied to 3-D geometries with time-varying boundary conditions such as those associated with pressurized thermal shock. Several investigators [3-5] have focused on an alternative superposition technique that requires calculation of a set of influence coefficients for a given 3-D crack model. In this technique, the crack surface of a specified geometry is subjected to four stress distributions in the radial direction: uniform, linear, quadratic, and cubic. Stress-intensity-factor influence coefficients (SIFICs) obtained from these solutions are then superimposed to obtain mode-I stress-intensity factors for other stress distributions, such as those due to internal pressure or thermal shock. Considerable economy can be achieved in this approach because the only direct 3-D solutions required are those used to calculate the influence coefficients.

The Heavy-Section Steel Technology Program at the Oak Ridge National Laboratory currently has the objective of developing a data base of validated SIFICs for a wide range of axially and circumferentially oriented finite-length semielliptical inner-surface flaw geometries for a range of clad-vessel geometries. The incorporation of this SIFIC data base into fracture mechanics codes such as FAVOR [6] will facilitate the generation of fracture mechanics solutions for a wide range of flaw geometries as may be required in structural integrity assessments of pressurized-water and boiling-water reactors.

In this paper, SIFICs are presented for axially and circumferentially oriented finitelength semielliptical inner-surface flaws with aspect ratios [total crack length (2c) to crack depth (a)] of 2,6, and 10 for clad cylinders having an internal radius $\left(\mathrm{R}_{\mathrm{i}}\right)$ to wall thickness ( $t$ ) ratio of 10. The SIFICs are computed for flaw depths in the range of $0.01 \leq \mathrm{a} / \mathrm{t} \leq 0.5$, with particular emphasis on shallow flaws $(\mathrm{a} / \mathrm{t} \leq 0.1)$ and cladding. SIFICs are computed for two cladding thicknesses $(t=3.96$ and $6.35 \mathrm{~mm})$. This paper first outlines the technique for computing the SIFICs. Next, results from the application of the superposition technique are compared with direct 3-D finite-element solutions. Finally, the calculated SIFICs are compared with results obtained by other investigators for $a / t \geq 0.2$ (SIFICs for shallow flaws from other sources were not available for comparison).

\section{SUPERPOSITION TECHNIQUE}

The stress-intensity factor $\left(\mathrm{K}_{\mathrm{I}}\right)$ is calculated by a superposition technique proposed by Bueckner [7]. Instead of calculating $\mathrm{K}_{I}$ for the cracked structure using the actual loads, the calculation is performed with a distributed pressure applied to the crack surfaces only. This pressure is opposite in sign, but equal in magnitude and distribution, to the stresses along the crack line that are calculated for the uncracked structure with the actual loads applied. For an arbitrary stress distribution and the case of a 3-D semielliptical flaw, the truncated stress distribution is approximated with a third-order polynomial as indicated by Eq. (1):

$$
\sigma\left(a^{\prime}\right)=C_{0}+C_{1}\left(a^{\prime} / a\right)+C_{2}\left(a^{\prime} / a\right)^{2}+C_{3}\left(a^{\prime} / a\right)^{3}
$$

where $\sigma\left(a^{\prime}\right)$ is the stress normal to the crack plane at radial position $a^{\prime}$, and $a^{\prime}$ and a are defined in Fig. 1. The $\mathrm{K}_{\mathrm{I}}$ values are calculated for each of the individual terms (stress distributions) in Eq. (1) and are then added to obtain the total $\mathrm{K}_{\mathrm{I}}$ value as indicated by Eq. (2):

$$
\mathrm{K}_{\mathrm{I}}(\mathrm{a})=\sum_{\mathrm{j}=0}^{3} \mathrm{~K}_{\mathrm{Ij}}(\mathrm{a})=\sum_{\mathrm{j}=0}^{3} \mathrm{C}_{\mathrm{j}} \sqrt{\pi \mathrm{a}} \mathrm{K}_{\mathrm{j}}^{*}(\mathrm{a})
$$

where 


$$
K_{j}^{*}(a)=K_{I j}^{\prime}(a) /\left(C_{j}^{\prime} \sqrt{\pi a}\right)
$$

Values of $\mathrm{K}_{\mathrm{Ij}}^{\prime}(\mathrm{a}) / \mathrm{C}_{\mathrm{j}}^{\prime}$ are calculated for each of the normalized stress distributions corresponding to each term in Eq. (1) (uniform, linear, quadratic, and cubic), using the 3-D finite-element analysis and an arbitrary value of $C_{j}^{\prime}$, such as unity. The quantity $K_{j}^{*}(a)$ is referred to as the influence coefficient and, as indicated by Eq. (3), is dimensionless. Once the influence coefficients are obtained, they can be used with any values of $C_{j}$ to obtain corresponding values of $\mathrm{K}_{\mathrm{I}}(\mathrm{a})$. For 3 -D flaws, $\mathrm{K}_{\mathrm{j}}^{*}(\mathrm{a})$ values can be calculated for several points along the crack front, in which case $K_{j}^{*}(a)$ in $E q$. (2) becomes $K_{j}^{*}(a, \phi)$, where $\phi$ is the elliptical angle denoting the point on the crack front.

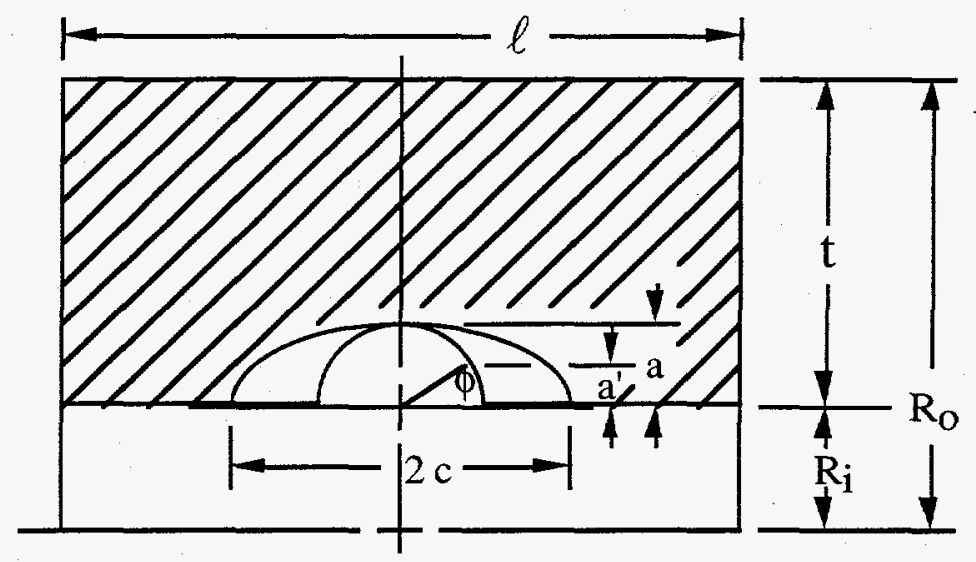

FIG. 1--Axially oriented semielliptical flaw on inner surface of cylinder.

The presence of a thin layer of stainless steel cladding on the inner surface of reactor pressure vessels has a significant effect on the $\mathrm{K}_{\mathrm{I}}$ values for inner-surface flaws because of very high thermal stresses generated in the cladding during a thermal transient. When using coefficients for 3-D flaws, it is necessary to represent the stress distribution in the uncracked cylinder with a third-order polynomial, and thus the discontinuity in the thermal stress at the clad-base material interface presents a problem. To accommodate the stress discontinuity associated with the cladding [5], influence coefficients were calculated for the cladding stresses alone; the corresponding $\mathrm{K}_{\mathrm{I}}$ value can then be superimposed on the $\mathrm{K}_{\mathrm{I}}$ value due to the stresses in the base material. This is accomplished by first calculating a $\mathrm{K}_{I}$ value for a continuous-function stress distribution obtained by a linear extrapolation of the stress distribution in the base material into the cladding. Then a $\mathrm{K}_{\mathrm{I}}$ value is calculated for a stress distribution in the cladding which is obtained by subtracting the extrapolated distribution from the actual distribution in the cladding, which is also assumed to be linear. The total $\mathrm{K}_{\mathrm{I}}$ value is simply the sum of the two. Because the stress distribution in the cladding is essentially linear, only a first-order polynomial is used for the cladding coefficients.

\section{CALCULATION OF SIFICs $\left(K_{\mathbf{j}}^{*}\right)$}

For the present study, $\mathrm{K}_{\mathrm{j}}^{*}$ values were computed using the ABAQUS [8] finiteelement code. ABAQUS is a nuclear-quality-assurance certified (NQA-1) code that 
employs a domain integral method for the computation of the J-integral. A 3-D finiteelement model was generated for each crack depth and aspect ratio. The analysis matrix included a crack depth range from $2.16 \mathrm{~mm}$ to $108.0 \mathrm{~mm}$ (crack depth/vessel thickness ratio of 0.01 to 0.5 ) and three different aspect ratios (2c/a) of 2, 6, and 10. A total of 39 finite-element models and over 200 analyses were required to generate the $K_{j}^{*}$ values.

The 3-D finite-element models were generated with the ORMGEN [9] mesh generating program. A typical finite-element mesh for an axial, inner-surface, semielliptical flaw is shown in Fig. $2(2 \mathrm{c} / \mathrm{a}=6$ and $\mathrm{a} / \mathrm{t}=0.2)$. The vessel in Fig. 2 has a $\mathrm{t}=216 \mathrm{~mm}$ (including cladding), $\mathrm{R}_{\mathrm{i}}=2184 \mathrm{~mm}$, and $\ell=4699 \mathrm{~mm}$.

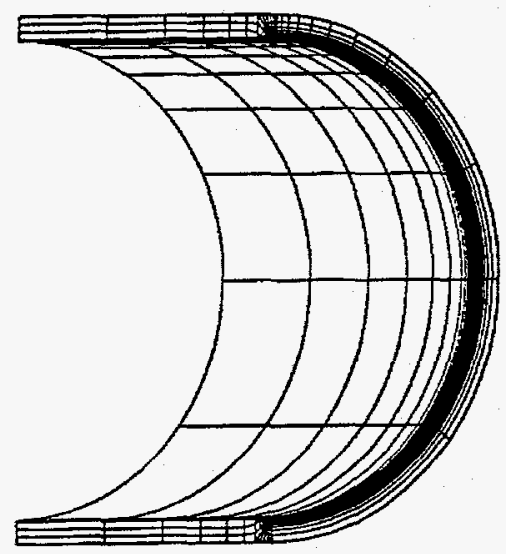

(a)

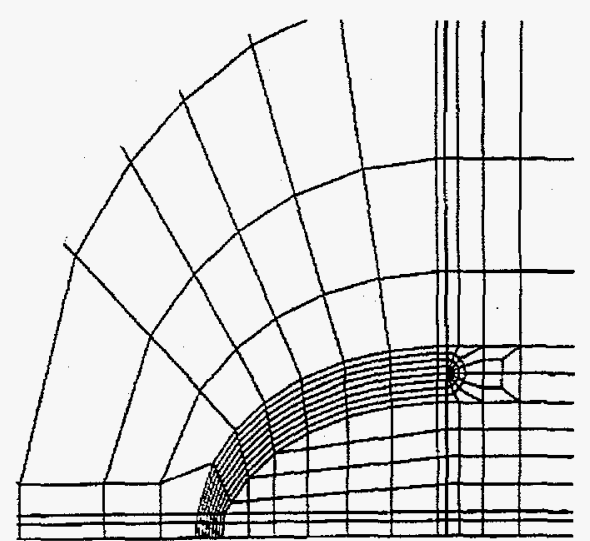

(b)

FIG. 2--Finite-element model for an axial inner-surface flaw having $R_{\mathrm{i}} / t=10, \mathrm{a} / \mathrm{t}=0.2$, and $2 \mathrm{c} / \mathrm{a}=6$ : (a) complete model, and (b) crack-tip region.

This model has 10792 nodes and 2255 twenty-noded isoparametric elements (reduced $2 \times 2$ $\mathrm{x} 2$ integration). The mesh refinement was increased (more nodes and elements) near the crack tip for models with shallower flaws. From symmetry conditions, only one-fourth of the vessel is included in the finite-element model. Figure 3 shows the finite-element mesh for a circumferential flaw $(2 \mathrm{c} / \mathrm{a}=6$ and $\mathrm{a} / \mathrm{t}=0.3)$.

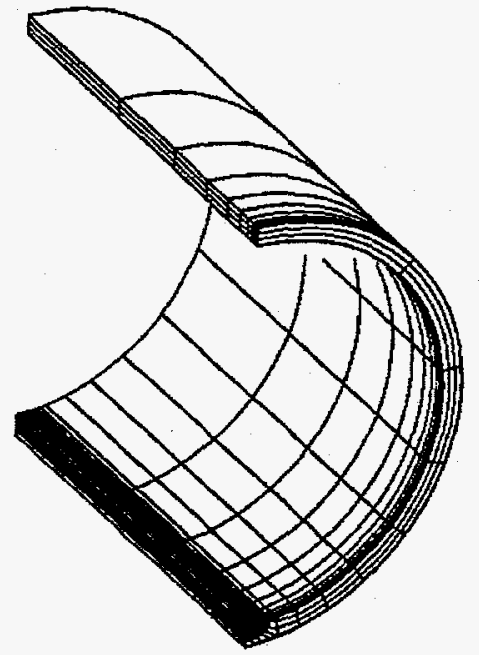

(a)

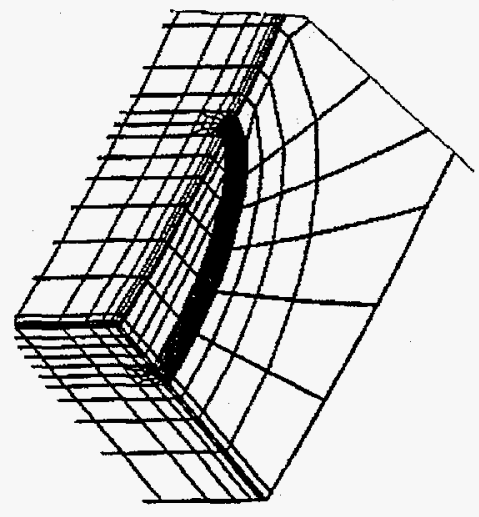

(b)

FIG. 3--Finite-element model for a circumferential inner-surface flaw having $R_{\mathrm{i}} / t=10$, $\mathrm{a} / \mathrm{t}=0.3$, and $2 \mathrm{c} / \mathrm{a}=6$ : (a) complete model, and (b) crack-tip region. 
In the process of calculating the SIFICs, careful attention was paid to using adequately converged finite-element meshes and an appropriate cylinder length. The number of elements in the circumferential and axial directions and around the crack front was increased, one at a time, to the point where addition of one element changed the value of $\mathrm{K}_{\mathrm{I}}$ by less than one percent. The resultant converged meshes for a $180^{\circ}$, half-length segment of the cylinder had $~ 30,000$ degrees of freedom. The minimum length of the cylinder to negate end effects was estimated from the cylinder radial dimensions [10] to be $\approx 3353 \mathrm{~mm}$. From previous studies [5], the appropriate length for a cylinder with a $6: 1$, inner-surface, semielliptical flaw $(\mathrm{a} / \mathrm{t}=0.2)$ was 1.4 times the estimated value, so a cylinder length of $4699 \mathrm{~mm}$ was used in these analyses. A second analysis was performed on a cylinder in which the length of the cylinder was doubled. The $\mathrm{K}_{I}$ values around the crack front changed less than one percent.

The adequacy of using SIFICs was investigated by calculating $\mathrm{K}_{\mathrm{I}}$ values by both the superposition (as implemented in the FAVOR code) and direct ABAQUS finite-element techniques. The first comparison was for the $6: 1$ axial semielliptical flaw with a/t $=0.075$ and the thermal loading through the vessel wall as indicated in Fig. 4 (a). Representative material properties for these analyses (Table 1) were obtained from the NRC-EPRI cosponsored PTS Benchmarking Exercise [11]. The results of this comparison analysis indicate a maximum difference of $\approx 2 \%$ along the crack front [Fig. 4 (b)]. The second comparison was for a 10:1 axial semi-elliptical flaw with $a / t=0.5$ and the same loading. The results, given in Fig. 4 (b), show good agreement.

TABLE 1--Material properties for cylinder analyses.

\begin{tabular}{lll}
\hline Material Property & Base & Clad \\
\hline Thermal Conductivity $(\mathrm{k}), \mathrm{W} / \mathrm{m} \cdot \mathrm{K}$ & 41.54 & 17.31 \\
Specific Heat $\left(\mathrm{c}_{\mathrm{p}}\right), \mathrm{J} / \mathrm{kg} \cdot \mathrm{K}$ & 502.4 & 502.4 \\
Density $(\rho), \mathrm{kg} / \mathrm{m}^{3}$ & 7833 & 7833 \\
Young's Modulus $(\mathrm{E}), \mathrm{GPa}$ & 193.05 & 186.16 \\
Coeff. of Thermal Exp. $(\alpha), \rho^{\circ} \mathrm{C}$ & $1.449 \mathrm{E}-05$ & $1.638 \mathrm{E}-05$ \\
Poisson's Ratio $(\mathrm{v})$ & 0.30 & 0.30 \\
Fluid-film heat transfer coefficient $(\mathrm{h})=1816 \mathrm{~W} / \mathrm{m}^{2} \cdot \mathrm{K}$ & \\
\hline
\end{tabular}
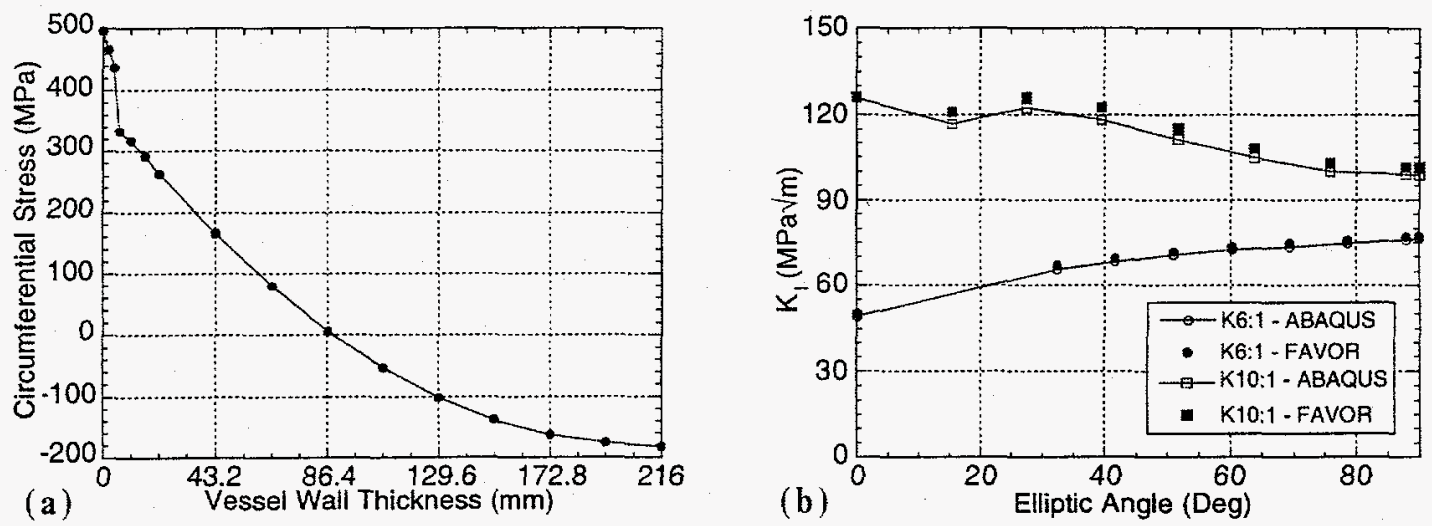

FIG. 4--(a)Thermal-stress profile in clad cylinder used to calculate $\mathrm{K}_{\mathrm{I}}$, and (b) comparison of $K_{I}$ values calculated by superposition and direct finite-element techniques for axially oriented 6:1 and 10:1 flaws ( $\mathrm{a} / \mathrm{t}=0.075$ and 0.5 , respectively) for severe thermal transient. 
Two analyses were performed on a clad cylinder having $R_{i} / t=10$ and $a / t=0.1$ with uniform and quadratic loading so a comparison of SIFICs could be made for vessels with the same $\mathrm{R}_{\mathrm{i}} / \mathrm{t}$ and $\mathrm{a} / \mathrm{t}$ ratios but different $\mathrm{t}$. The SIFICs around the crack front had less than $0.02 \%$ difference between the analyses of the two cylinders $(t=216 \mathrm{~mm}$ and $108 \mathrm{~mm})$.

At the outset of the present study, it was anticipated that SIFICs for shallow circumferential flaws ( $a / t \leq 0.1)$ would be essentially identical to those computed for shallow axial flaws. This anticipated result was arrived at by examining SIFICs computed and reported $[12,13]$ previously for 2-D flaws. Figure 5 shows the maximum thermally induced value of $\mathrm{K}_{\mathrm{I}}$ (with respect to time) as a function of crack depth for both long axial flaws and continuous circumferential flaws for a reference calculational model used in the NRC-EPRI cosponsored PTS Benchmarking Exercise [11]. It can be seen that the two

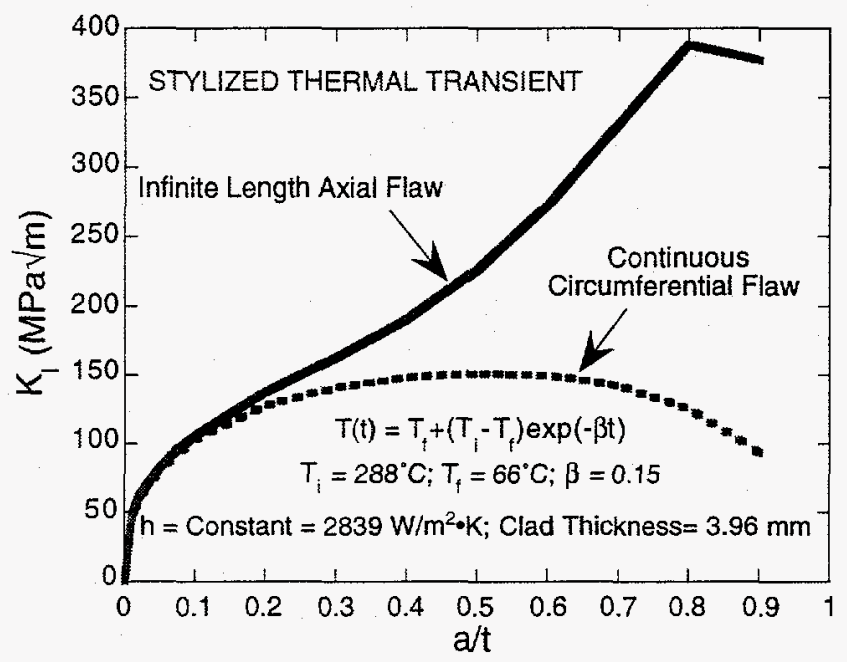

FIG. 5--Variation of maximum $\mathrm{K}_{\mathrm{I}}$ (with respect to time) as a function of crack depth for inner-surface long axial flaws and continuous circumferential flaws for the stylized thermal transient reference calculational model.

flaw geometries give essentially identical results for $\mathrm{a} / \mathrm{t} \leq 0.1$, however, significant differences can be observed for deeper flaws. It could be expected that finite axial and circumferential flaws would show no differences for shallow flaws and less difference than that shown in Fig. 5 for deeper flaws due to the increased constraint provided by the 3-D geometry.

These expected results were indeed realized in the present study as can be observed in Fig. 6. Variations of the SIFICs at the deepest point on the crack front are shown as a function of $a / t$ ratios for $2 \mathrm{c} / \mathrm{a}=10$. It can be observed that there are essentially no differences between calculated SIFICs for circumferential and axial semielliptical innersurface flaws having an a/t $\leq 0.3$. Small differences $(\sim 5 \%)$ can be observed for flaw geometries having an $a / t=0.5$ and $2 c / a=10$. This difference is less for $2 c / a$ of 2 and 6 . Calculations for shallow flaws indicated no differences between SIFIC for finite axial and circumferential flaws.

Tables 2-10 give the results of this study in terms of non-dimensionalized $K_{j}^{*}$ values for axially and circumferentially oriented, inner-surface flaws in a cylinder, with surface length-to-depth ratios of $2: 1,6: 1$, and $10: 1$, and with $\mathrm{t}=216 \mathrm{~mm}$ (including the cladding), $\mathrm{R}_{\mathrm{i}}=2184 \mathrm{~mm}$, and $\ell=4699 \mathrm{~mm}$. Also, there are coefficients for two cladding thicknesses $\left(\mathrm{t}_{\mathrm{cl}}\right), 6.35 \mathrm{~mm}$ and $3.96 \mathrm{~mm} . \mathrm{K}_{\mathrm{j}}^{*}$ values are obtained by computing $\mathrm{K}$ from $\mathrm{J}$ using a plane-strain relation, where $\mathrm{K}$ is the stress-intensity factor per unit stress applied to 
the crack face and then dividing by $\sqrt{\pi \mathrm{a}}$. While English units were utilized to compute the $\mathrm{K}_{\mathrm{j}}^{*}$ values, the $\mathrm{K}_{\mathrm{j}}^{*}$ values, which are nondimensionalized, in the tables are appropriate for any consistent set of units (English, SI, etc.).
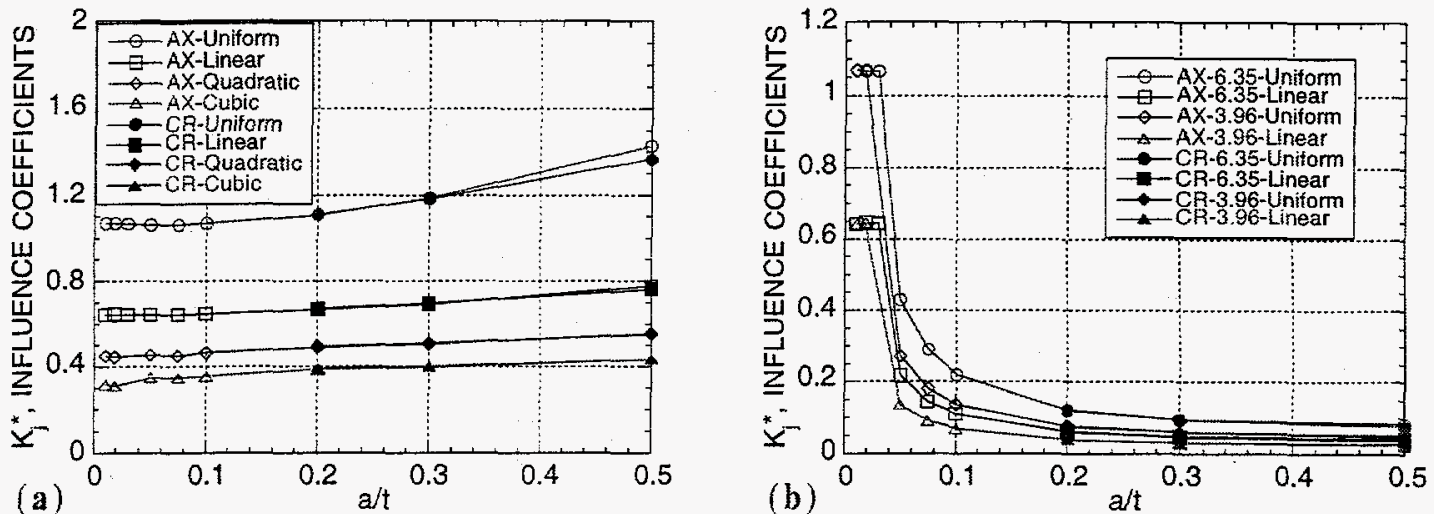

FIG. 6--Influence coefficients for semielliptical inner-surface flaws $(2 \mathrm{c} / \mathrm{a}=10)$ at $\phi=90^{\circ}$ : (a) four reference loads applied over crack face, and (b) uniform and linear load applied over portion of crack face within cladding.

TABLE 2--Influence coefficients for inside axial surface crack: $a / t=0.01$.

\begin{tabular}{|c|c|c|c|c|c|c|c|c|c|}
\hline $\begin{array}{l}\text { Aspect } \\
\text { Ratio }\end{array}$ & $\begin{array}{l}\text { Elliptic } \\
\text { Angle }\end{array}$ & $\begin{array}{l}\text { K0 } \\
\text { Uniform }\end{array}$ & $\begin{array}{l}\text { K1 } \\
\text { Linear }\end{array}$ & $\begin{array}{l}\text { K2 } \\
\text { Quadratic }\end{array}$ & $\begin{array}{l}\text { K3 } \\
\text { Cubic } \\
\end{array}$ & $\begin{array}{l}K 0 \\
t_{\mathrm{cl}}=6.35\end{array}$ & $\begin{array}{l}\mathrm{K} 1 \\
\mathrm{t}_{\mathrm{cl}}=6.35\end{array}$ & $\begin{array}{l}\mathrm{K} 0 \\
\mathrm{t}_{\mathrm{cl}}=3.96\end{array}$ & $\begin{array}{l}\mathrm{K} 1 \\
\mathrm{t}_{\mathrm{cl}}=3.96\end{array}$ \\
\hline \multirow[t]{9}{*}{$2: 1$} & 0.00 & 0.764 & 0.153 & 0.061 & 0.034 & 0.764 & 0.153 & 0.764 & 0.153 \\
\hline & 2.37 & 0.754 & 0.165 & 0.062 & 0.032 & 0.754 & 0.165 & 0.754 & 0.165 \\
\hline & 16.6 & 0.690 & 0.192 & 0.079 & 0.040 & 0.690 & 0.192 & 0.690 & 0.192 \\
\hline & 30.8 & 0.669 & 0.264 & 0.127 & 0.069 & 0.669 & 0.264 & 0.669 & 0.264 \\
\hline & 45.0 & 0.660 & 0.335 & 0.196 & 0.124 & 0.660 & 0.335 & 0.660 & 0.335 \\
\hline & 59.2 & 0.653 & 0.393 & 0.269 & 0.198 & 0.653 & 0.393 & 0.653 & 0.393 \\
\hline & 73.4 & 0.651 & 0.434 & 0.329 & 0.268 & 0.651 & 0.434 & 0.651 & 0.434 \\
\hline & 87.6 & 0.649 & 0.463 & 0.366 & 0.310 & 0.649 & 0.463 & 0.649 & 0.463 \\
\hline & 90.0 & 0.649 & 0.468 & 0.372 & 0.317 & 0.649 & 0.468 & 0.649 & 0.468 \\
\hline \multirow[t]{9}{*}{$6: 1$} & 0.00 & 0.670 & 0.134 & 0.048 & 0.024 & 0.670 & 0.134 & 0.670 & 0.134 \\
\hline & 2.37 & 0.667 & 0.134 & 0.043 & 0.019 & 0.667 & 0.134 & 0.667 & 0.134 \\
\hline & 16.6 & 0.654 & 0.170 & 0.055 & 0.009 & 0.654 & 0.170 & 0.654 & 0.170 \\
\hline & 30.8 & 0.741 & 0.269 & 0.109 & 0.029 & 0.741 & 0.269 & 0.741 & 0.269 \\
\hline & 45.0 & 0.827 & 0.381 & 0.199 & 0.100 & 0.827 & 0.381 & 0.827 & 0.381 \\
\hline & 59.2 & 0.893 & 0.481 & 0.302 & 0.197 & 0.893 & 0.481 & 0.893 & 0.481 \\
\hline & 73.4 & 0.938 & 0.559 & 0.389 & 0.290 & 0.938 & 0.559 & 0.938 & 0.559 \\
\hline & 87.6 & 0.970 & 0.594 & 0.435 & 0.341 & 0.970 & 0.594 & 0.970 & 0.594 \\
\hline & 90.0 & 0.975 & 0.601 & 0.443 & 0.350 & 0.975 & 0.601 & 0.975 & 0.601 \\
\hline \multirow[t]{9}{*}{$10: 1$} & 0.00 & 0.515 & 0.090 & 0.020 & 0.006 & 0.515 & 0.090 & 0.515 & 0.090 \\
\hline & 2.37 & 0.529 & 0.094 & 0.010 & 0.005 & 0.529 & 0.094 & 0.529 & 0.094 \\
\hline & 16.6 & 0.610 & 0.146 & 0.033 & 0.005 & 0.610 & 0.146 & 0.610 & 0.146 \\
\hline & 30.8 & 0.762 & 0.258 & 0.060 & 0.019 & 0.762 & 0.258 & 0.762 & 0.258 \\
\hline & 45.0 & 0.889 & 0.389 & 0.171 & 0.066 & 0.889 & 0.389 & 0.889 & 0.389 \\
\hline & 59.2 & 0.979 & 0.507 & 0.290 & 0.136 & 0.979 & 0.507 & 0.979 & 0.507 \\
\hline & 73.4 & 1.033 & 0.593 & 0.389 & 0.249 & 1.033 & 0.593 & 1.033 & 0.593 \\
\hline & 87.6 & 1.064 & 0.635 & 0.439 & 0.307 & 1.064 & 0.635 & 1.064 & 0.635 \\
\hline & 90.0 & 1.069 & 0.642 & 0.447 & 0.316 & 1.069 & 0.642 & 1.069 & 0.642 \\
\hline
\end{tabular}


TABLE 3--Influence coefficients for inside axial surface crack: $\mathrm{a} / \mathrm{t}=0.0184$.

\begin{tabular}{llllllllll}
\hline Aspect & Elliptic & K0 & K1 & K2 & K3 & K0 & K1 & K0 & K1 \\
Ratio & Angle & Uniform & Linear & Quadratic & Cubic & $t_{c l=6.35}$ & $t_{\mathrm{cl}=6.35}$ & $t_{\mathrm{cl}=3.96}$ & $t_{\mathrm{cl}=3.96}$ \\
\hline $2: 1$ & 0.00 & 0.777 & 0.155 & 0.061 & 0.034 & 0.777 & 0.155 & 0.777 & 0.155 \\
& 2.37 & 0.767 & 0.167 & 0.062 & 0.032 & 0.767 & 0.167 & 0.767 & 0.167 \\
& 16.6 & 0.700 & 0.194 & 0.079 & 0.040 & 0.700 & 0.194 & 0.700 & 0.194 \\
& 30.8 & 0.677 & 0.266 & 0.127 & 0.069 & 0.677 & 0.266 & 0.677 & 0.266 \\
& 45.0 & 0.667 & 0.338 & 0.196 & 0.125 & 0.667 & 0.338 & 0.667 & 0.338 \\
& 59.2 & 0.660 & 0.397 & 0.270 & 0.198 & 0.660 & 0.397 & 0.660 & 0.397 \\
& 73.4 & 0.657 & 0.438 & 0.330 & 0.267 & 0.657 & 0.438 & 0.657 & 0.438 \\
& 87.6 & 0.654 & 0.467 & 0.366 & 0.310 & 0.654 & 0.467 & 0.654 & 0.467 \\
& 90.0 & 0.653 & 0.472 & 0.373 & 0.317 & 0.653 & 0.472 & 0.653 & 0.472 \\
\hline $6: 1$ & 0.00 & 0.653 & 0.128 & 0.043 & 0.021 & 0.653 & 0.128 & 0.653 & 0.128 \\
& 2.37 & 0.654 & 0.128 & 0.038 & 0.016 & 0.654 & 0.128 & 0.654 & 0.128 \\
& 16.6 & 0.654 & 0.168 & 0.045 & 0.021 & 0.654 & 0.168 & 0.654 & 0.168 \\
& 30.8 & 0.758 & 0.271 & 0.099 & 0.026 & 0.758 & 0.271 & 0.758 & 0.271 \\
& 45.0 & 0.852 & 0.387 & 0.192 & 0.085 & 0.852 & 0.387 & 0.852 & 0.387 \\
& 59.2 & 0.920 & 0.492 & 0.298 & 0.187 & 0.920 & 0.492 & 0.920 & 0.492 \\
& 73.4 & 0.963 & 0.569 & 0.387 & 0.283 & 0.963 & 0.569 & 0.963 & 0.569 \\
& 87.6 & 0.994 & 0.609 & 0.434 & 0.335 & 0.994 & 0.609 & 0.994 & 0.609 \\
& 90.0 & 0.999 & 0.616 & 0.442 & 0.344 & 0.999 & 0.616 & 0.999 & 0.616 \\
\hline $10: 1$ & 0.00 & 0.525 & 0.092 & 0.019 & 0.007 & 0.525 & 0.092 & 0.525 & 0.092 \\
& 2.37 & 0.538 & 0.096 & 0.009 & 0.005 & 0.538 & 0.096 & 0.538 & 0.096 \\
& 16.6 & 0.621 & 0.149 & 0.039 & 0.005 & 0.621 & 0.149 & 0.621 & 0.149 \\
& 30.8 & 0.777 & 0.262 & 0.050 & 0.022 & 0.777 & 0.262 & 0.777 & 0.262 \\
& 45.0 & 0.899 & 0.392 & 0.164 & 0.075 & 0.899 & 0.392 & 0.899 & 0.392 \\
& 59.2 & 0.982 & 0.509 & 0.283 & 0.127 & 0.982 & 0.509 & 0.982 & 0.509 \\
& 73.4 & 1.033 & 0.595 & 0.383 & 0.242 & 1.033 & 0.595 & 1.033 & 0.595 \\
& 87.6 & 1.063 & 0.637 & 0.433 & 0.300 & 1.063 & 0.637 & 1.063 & 0.637 \\
& 90.0 & 1.068 & 0.644 & 0.441 & 0.310 & 1.068 & 0.644 & 1.068 & 0.644 \\
\hline
\end{tabular}

TABLE 4--Influence coefficients for inside axial surface crack: $\mathrm{a} / \mathrm{t}=0.05$.

\begin{tabular}{llllllllll}
\hline $\begin{array}{l}\text { Aspect } \\
\text { Ratio }\end{array}$ & $\begin{array}{l}\text { Elliptic } \\
\text { Angle }\end{array}$ & $\begin{array}{l}\text { K0 } \\
\text { Uniform }\end{array}$ & $\begin{array}{l}\text { K1 } \\
\text { Linear }\end{array}$ & $\begin{array}{l}\mathrm{K} 2 \\
\text { Quadratic }\end{array}$ & $\begin{array}{l}\mathrm{K} 3 \\
\text { Cubic }\end{array}$ & $\begin{array}{l}\mathrm{K} 0 \\
\mathrm{t}_{\mathrm{cl}}=6.35\end{array}$ & $\begin{array}{l}\mathrm{K} 1 \\
\mathrm{t}_{\mathrm{cl}}=6.35\end{array}$ & $\begin{array}{l}\mathrm{K} 0 \\
\mathrm{t}_{\mathrm{cl}}=3.96\end{array}$ & $\begin{array}{l}\mathrm{K} 1 \\
\mathrm{t}_{\mathrm{cl}}=3.96\end{array}$ \\
\hline $2: 1$ & 0.00 & 0.701 & 0.133 & 0.045 & 0.022 & 0.596 & 0.171 & 0.598 & 0.212 \\
& 31.0 & 0.681 & 0.276 & 0.131 & 0.070 & 0.615 & 0.278 & 0.238 & 0.119 \\
& 40.5 & 0.670 & 0.323 & 0.178 & 0.108 & 0.386 & 0.208 & 0.175 & 0.090 \\
& 50.0 & 0.663 & 0.367 & 0.231 & 0.158 & 0.268 & 0.147 & 0.146 & 0.075 \\
& 59.4 & 0.659 & 0.404 & 0.282 & 0.212 & 0.226 & 0.124 & 0.129 & 0.067 \\
& 68.9 & 0.654 & 0.432 & 0.325 & 0.263 & 0.206 & 0.112 & 0.119 & 0.062 \\
& 78.4 & 0.653 & 0.451 & 0.355 & 0.301 & 0.194 & 0.105 & 0.114 & 0.059 \\
& 87.9 & 0.651 & 0.472 & 0.379 & 0.327 & 0.186 & 0.101 & 0.108 & 0.056 \\
& 90.0 & 0.651 & 0.476 & 0.384 & 0.333 & 0.184 & 0.100 & 0.107 & 0.056 \\
\hline $6: 1$ & 0.00 & 0.513 & 0.100 & 0.027 & 0.010 & 0.479 & 0.186 & 0.444 & 0.177 \\
& 31.0 & 0.764 & 0.275 & 0.100 & 0.022 & 0.700 & 0.346 & 0.336 & 0.166 \\
& 40.5 & 0.823 & 0.352 & 0.162 & 0.072 & 0.528 & 0.270 & 0.292 & 0.147 \\
& 50.0 & 0.875 & 0.429 & 0.235 & 0.137 & 0.445 & 0.232 & 0.271 & 0.137 \\
& 59.4 & 0.915 & 0.497 & 0.310 & 0.210 & 0.417 & 0.215 & 0.259 & 0.130 \\
& 68.9 & 0.944 & 0.551 & 0.375 & 0.279 & 0.400 & 0.205 & 0.251 & 0.126 \\
& 78.4 & 0.964 & 0.589 & 0.423 & 0.334 & 0.391 & 0.199 & 0.247 & 0.124 \\
& 87.9 & 0.984 & 0.611 & 0.449 & 0.363 & 0.384 & 0.195 & 0.243 & 0.122 \\
& 90.0 & 0.989 & 0.616 & 0.454 & 0.369 & 0.382 & 0.194 & 0.242 & 0.122 \\
\hline $10: 1$ & 0.00 & 0.424 & 0.075 & 0.012 & 0.008 & 0.373 & 0.163 & 0.323 & 0.147 \\
& 31.0 & 0.781 & 0.261 & 0.044 & 0.016 & 0.713 & 0.345 & 0.372 & 0.182 \\
& 40.5 & 0.868 & 0.350 & 0.126 & 0.056 & 0.578 & 0.300 & 0.333 & 0.167 \\
& 50.0 & 0.932 & 0.437 & 0.211 & 0.082 & 0.501 & 0.258 & 0.309 & 0.155 \\
& 59.4 & 0.981 & 0.514 & 0.294 & 0.172 & 0.471 & 0.240 & 0.293 & 0.147 \\
& 68.9 & 1.017 & 0.575 & 0.367 & 0.251 & 0.450 & 0.229 & 0.283 & 0.142 \\
& 78.4 & 1.040 & 0.617 & 0.419 & 0.311 & 0.440 & 0.222 & 0.278 & 0.139 \\
& 87.9 & 1.057 & 0.639 & 0.446 & 0.341 & 0.431 & 0.217 & 0.272 & 0.136 \\
& 90.0 & 1.061 & 0.644 & 0.452 & 0.347 & 0.430 & 0.216 & 0.271 & 0.136 \\
\hline
\end{tabular}


TABLE 5--Influence coefficients for inside axial surface crack: $\mathrm{a} / \mathrm{t}=0.075$.

\begin{tabular}{|c|c|c|c|c|c|c|c|c|c|}
\hline $\begin{array}{l}\text { Aspect } \\
\text { Ratio }\end{array}$ & $\begin{array}{l}\text { Elliptic } \\
\text { Angle }\end{array}$ & $\begin{array}{l}\text { K0 } \\
\text { Uniform }\end{array}$ & $\begin{array}{l}\text { K1 } \\
\text { Linear }\end{array}$ & $\begin{array}{l}\text { K2 } \\
\text { Quadratic }\end{array}$ & $\begin{array}{l}\text { K3 } \\
\text { Cubic }\end{array}$ & $\begin{array}{l}\mathrm{K} 0 \\
\mathrm{t}_{\mathrm{cl}}=6.35\end{array}$ & $\begin{array}{l}\mathrm{K} 1 \\
\mathrm{t}_{\mathrm{cl}}=6.35\end{array}$ & $\begin{array}{l}\mathrm{K} 0 \\
t_{\mathrm{cl}}=3.96\end{array}$ & $\begin{array}{l}\mathrm{K} 1 \\
\mathrm{t}_{\mathrm{cl}}=3.96\end{array}$ \\
\hline \multirow[t]{9}{*}{$2: 1$} & 0.00 & 0.697 & 0.134 & 0.045 & 0.022 & 0.604 & 0.210 & 0.511 & 0.204 \\
\hline & 32.3 & 0.683 & 0.284 & 0.137 & 0.075 & 0.250 & 0.127 & 0.138 & 0.063 \\
\hline & 41.6 & 0.673 & 0.331 & 0.185 & 0.114 & 0.187 & 0.096 & 0.108 & 0.054 \\
\hline & 50.9 & 0.666 & 0.373 & 0.236 & 0.163 & 0.157 & 0.081 & 0.092 & 0.047 \\
\hline & 60.1 & 0.662 & 0.409 & 0.286 & 0.217 & 0.139 & 0.073 & 0.083 & 0.042 \\
\hline & 69.4 & 0.657 & 0.436 & 0.327 & 0.266 & 0.129 & 0.067 & 0.076 & 0.039 \\
\hline & 78.6 & 0.656 & 0.454 & 0.356 & 0.303 & 0.123 & 0.064 & 0.073 & 0.037 \\
\hline & 87.9 & 0.654 & 0.474 & 0.379 & 0.328 & 0.118 & 0.061 & 0.070 & 0.036 \\
\hline & 90.0 & 0.654 & 0.478 & 0.384 & 0.334 & 0.116 & 0.060 & 0.069 & 0.035 \\
\hline \multirow[t]{9}{*}{$6: 1$} & 0.00 & 0.499 & 0.099 & 0.026 & 0.009 & 0.436 & 0.171 & 0.357 & 0.162 \\
\hline & 32.3 & 0.772 & 0.285 & 0.102 & 0.018 & 0.356 & 0.177 & 0.208 & 0.099 \\
\hline & 41.6 & 0.829 & 0.360 & 0.164 & 0.073 & 0.311 & 0.157 & 0.188 & 0.093 \\
\hline & 50.9 & 0.878 & 0.434 & 0.237 & 0.138 & 0.289 & 0.146 & 0.177 & 0.089 \\
\hline & 60.1 & 0.916 & 0.499 & 0.309 & 0.210 & 0.276 & 0.139 & 0.170 & 0.085 \\
\hline & 69.4 & 0.943 & 0.551 & 0.372 & 0.278 & 0.268 & 0.135 & 0.166 & 0.083 \\
\hline & 78.6 & 0.963 & 0.587 & 0.418 & 0.330 & 0.264 & 0.133 & 0.163 & 0.082 \\
\hline & 87.9 & 0.982 & 0.609 & 0.443 & 0.358 & 0.266 & 0.134 & 0.165 & 0.082 \\
\hline & 90.0 & 0.986 & 0.614 & 0.448 & 0.365 & 0.267 & 0.134 & 0.165 & 0.082 \\
\hline \multirow[t]{9}{*}{$10: 1$} & 0.00 & 0.368 & 0.077 & 0.011 & 0.010 & 0.312 & 0.141 & 0.231 & 0.127 \\
\hline & 32.3 & 0.796 & 0.276 & 0.049 & 0.025 & 0.394 & 0.195 & 0.234 & 0.112 \\
\hline & 41.6 & 0.878 & 0.363 & 0.131 & 0.053 & 0.353 & 0.178 & 0.216 & 0.107 \\
\hline & 50.9 & 0.939 & 0.447 & 0.215 & 0.089 & 0.328 & 0.165 & 0.203 & 0.101 \\
\hline & 60.1 & 0.985 & 0.520 & 0.296 & 0.176 & 0.312 & 0.157 & 0.194 & 0.097 \\
\hline & 69.4 & 1.018 & 0.578 & 0.365 & 0.252 & 0.302 & 0.152 & 0.188 & 0.094 \\
\hline & 78.6 & 1.039 & 0.618 & 0.415 & 0.310 & 0.296 & 0.149 & 0.184 & 0.092 \\
\hline & 87.9 & 1.056 & 0.639 & 0.441 & 0.339 & 0.291 & 0.146 & 0.181 & 0.090 \\
\hline & 90.0 & 1.060 & 0.644 & 0.447 & 0.345 & 0.289 & 0.145 & 0.180 & 0.090 \\
\hline
\end{tabular}

TABLE 6--Influence coefficients for inside axial surface crack: $a / t=0.1$.

\begin{tabular}{|c|c|c|c|c|c|c|c|c|c|}
\hline $\begin{array}{l}\text { Aspect } \\
\text { Ratio }\end{array}$ & $\begin{array}{l}\text { Elliptic } \\
\text { Angle }\end{array}$ & $\begin{array}{l}\text { Ko } \\
\text { Uniform }\end{array}$ & $\begin{array}{l}\text { K1 } \\
\text { Linear }\end{array}$ & $\begin{array}{l}\text { K2 } \\
\text { Quadratic }\end{array}$ & $\begin{array}{l}\text { K3 } \\
\text { Cubic } \\
\end{array}$ & $\begin{array}{l}\mathrm{K} 0 \\
\mathrm{t}_{\mathrm{cl}}=6.35\end{array}$ & $\begin{array}{l}\mathrm{K} 1 \\
t_{\mathrm{cl}}=6.35\end{array}$ & $\begin{array}{l}\mathrm{K} 0 \\
\mathrm{t}_{\mathrm{cl}}=3.96\end{array}$ & $\begin{array}{l}\mathrm{K} 1 \\
\mathrm{t}_{\mathrm{cl}}=3.96\end{array}$ \\
\hline \multirow[t]{9}{*}{$2: 1$} & 0.00 & 0.715 & 0.133 & 0.046 & 0.023 & 0.576 & 0.217 & 0.486 & 0.211 \\
\hline & 27.2 & 0.687 & 0.256 & 0.116 & 0.060 & 0.206 & 0.102 & 0.116 & 0.052 \\
\hline & 37.3 & 0.676 & 0.308 & 0.164 & 0.095 & 0.146 & 0.074 & 0.087 & 0.042 \\
\hline & 47.4 & 0.669 & 0.357 & 0.220 & 0.145 & 0.119 & 0.061 & 0.072 & 0.036 \\
\hline & 57.5 & 0.663 & 0.399 & 0.275 & 0.203 & 0.104 & 0.053 & 0.063 & 0.032 \\
\hline & 67.7 & 0.659 & 0.431 & 0.323 & 0.258 & 0.095 & 0.049 & 0.057 & 0.029 \\
\hline & 77.8 & 0.657 & 0.452 & 0.357 & 0.301 & 0.090 & 0.046 & 0.055 & 0.028 \\
\hline & 87.9 & 0.655 & 0.474 & 0.383 & 0.329 & 0.086 & 0.044 & 0.052 & 0.026 \\
\hline & 90.0 & 0.655 & 0.479 & 0.388 & 0.335 & 0.085 & 0.043 & 0.051 & 0.026 \\
\hline \multirow[t]{9}{*}{$6: 1$} & 0.00 & 0.553 & 0.095 & 0.027 & 0.011 & 0.457 & 0.179 & 0.380 & 0.170 \\
\hline & 27.2 & 0.741 & 0.242 & 0.084 & 0.011 & 0.278 & 0.137 & 0.164 & 0.079 \\
\hline & 37.3 & 0.811 & 0.324 & 0.144 & 0.053 & 0.240 & 0.120 & 0.147 & 0.073 \\
\hline & 47.4 & 0.869 & 0.407 & 0.220 & 0.118 & 0.221 & 0.111 & 0.136 & 0.068 \\
\hline & 57.5 & 0.915 & 0.483 & 0.299 & 0.194 & 0.210 & 0.105 & 0.130 & 0.065 \\
\hline & 67.7 & 0.948 & 0.544 & 0.371 & 0.270 & 0.203 & 0.102 & 0.126 & 0.063 \\
\hline & 77.8 & 0.971 & 0.586 & 0.424 & 0.330 & 0.199 & 0.100 & 0.124 & 0.062 \\
\hline & 87.9 & 0.991 & 0.610 & 0.453 & 0.362 & 0.196 & 0.098 & 0.122 & 0.061 \\
\hline & 90.0 & 0.996 & 0.615 & 0.459 & 0.369 & 0.195 & 0.098 & 0.121 & 0.061 \\
\hline \multirow[t]{9}{*}{$10: 1$} & 0.00 & 0.417 & 0.071 & 0.013 & 0.006 & 0.352 & 0.156 & 0.286 & 0.144 \\
\hline & 27.2 & 0.752 & 0.228 & 0.041 & 0.017 & 0.310 & 0.151 & 0.186 & 0.089 \\
\hline & 37.3 & 0.849 & 0.321 & 0.116 & 0.057 & 0.274 & 0.137 & 0.169 & 0.084 \\
\hline & 47.4 & 0.922 & 0.415 & 0.202 & 0.067 & 0.252 & 0.126 & 0.157 & 0.078 \\
\hline & 57.5 & 0.979 & 0.500 & 0.290 & 0.162 & 0.239 & 0.119 & 0.149 & 0.074 \\
\hline & 67.7 & 1.020 & 0.569 & 0.370 & 0.247 & 0.230 & 0.115 & 0.143 & 0.072 \\
\hline & 77.8 & 1.046 & 0.616 & 0.428 & 0.313 & 0.225 & 0.113 & 0.140 & 0.070 \\
\hline & 87.9 & 1.065 & 0.640 & 0.457 & 0.346 & 0.220 & 0.110 & 0.137 & 0.069 \\
\hline & 90.0 & 1.068 & 0.645 & 0.463 & 0.353 & 0.219 & 0.110 & 0.137 & 0.068 \\
\hline
\end{tabular}


TABLE 7--Influence coefficients for inside axial surface crack: $\mathrm{a} / \mathrm{t}=0.2$.

\begin{tabular}{llllllllll}
\hline $\begin{array}{l}\text { Aspect } \\
\text { Ratio }\end{array}$ & Elliptic & K0 & $\mathrm{K} 1$ & $\mathrm{~K} 2$ & $\mathrm{~K} 3$ & $\mathrm{~K} 0$ & $\mathrm{~K} 1$ & $\mathrm{~K} 0$ & $\mathrm{~K} 1$ \\
\hline $2: 1$ & 0.00 & 0.723 & 0.127 & 0.046 & 0.025 & 0.471 & 0.204 & 0.391 & 0.195 \\
& 19.8 & 0.695 & 0.214 & 0.089 & 0.044 & 0.130 & 0.063 & 0.071 & 0.031 \\
& 31.1 & 0.679 & 0.273 & 0.133 & 0.073 & 0.080 & 0.039 & 0.048 & 0.023 \\
& 42.5 & 0.671 & 0.332 & 0.192 & 0.120 & 0.061 & 0.031 & 0.038 & 0.019 \\
& 53.8 & 0.665 & 0.383 & 0.255 & 0.182 & 0.052 & 0.026 & 0.032 & 0.016 \\
& 65.2 & 0.660 & 0.423 & 0.312 & 0.245 & 0.047 & 0.023 & 0.029 & 0.014 \\
& 76.5 & 0.658 & 0.450 & 0.354 & 0.296 & 0.044 & 0.022 & 0.027 & 0.014 \\
& 87.9 & 0.656 & 0.475 & 0.384 & 0.329 & 0.041 & 0.021 & 0.025 & 0.013 \\
& 90.0 & 0.656 & 0.479 & 0.389 & 0.335 & 0.040 & 0.020 & 0.025 & 0.013 \\
\hline $6: 1$ & 0.00 & 0.625 & 0.101 & 0.034 & 0.017 & 0.423 & 0.189 & 0.354 & 0.179 \\
& 19.8 & 0.699 & 0.194 & 0.066 & 0.019 & 0.157 & 0.074 & 0.093 & 0.043 \\
& 31.1 & 0.781 & 0.280 & 0.118 & 0.045 & 0.127 & 0.063 & 0.079 & 0.039 \\
& 42.5 & 0.856 & 0.375 & 0.195 & 0.101 & 0.116 & 0.058 & 0.072 & 0.036 \\
& 53.8 & 0.915 & 0.464 & 0.283 & 0.180 & 0.110 & 0.055 & 0.069 & 0.034 \\
& 65.2 & 0.958 & 0.538 & 0.366 & 0.265 & 0.106 & 0.053 & 0.066 & 0.033 \\
& 76.5 & 0.986 & 0.590 & 0.430 & 0.336 & 0.104 & 0.052 & 0.065 & 0.032 \\
& 87.9 & 1.010 & 0.619 & 0.464 & 0.373 & 0.102 & 0.051 & 0.064 & 0.032 \\
& 90.0 & 1.014 & 0.624 & 0.470 & 0.380 & 0.102 & 0.051 & 0.063 & 0.032 \\
\hline $10: 1$ & 0.00 & 0.506 & 0.077 & 0.022 & 0.009 & 0.368 & 0.180 & 0.314 & 0.164 \\
& 19.8 & 0.694 & 0.183 & 0.050 & 0.025 & 0.174 & 0.078 & 0.106 & 0.050 \\
& 31.1 & 0.815 & 0.280 & 0.107 & 0.011 & 0.149 & 0.073 & 0.093 & 0.046 \\
& 42.5 & 0.915 & 0.387 & 0.190 & 0.083 & 0.137 & 0.068 & 0.085 & 0.043 \\
& 53.8 & 0.991 & 0.488 & 0.287 & 0.170 & 0.130 & 0.065 & 0.081 & 0.040 \\
& 65.2 & 1.045 & 0.572 & 0.379 & 0.263 & 0.125 & 0.062 & 0.078 & 0.039 \\
& 76.5 & 1.080 & 0.631 & 0.449 & 0.340 & 0.122 & 0.061 & 0.077 & 0.038 \\
& 87.9 & 1.103 & 0.660 & 0.483 & 0.378 & 0.120 & 0.060 & 0.075 & 0.037 \\
& 90.0 & 1.107 & 0.666 & 0.490 & 0.385 & 0.120 & 0.060 & 0.075 & 0.037 \\
\hline
\end{tabular}

TABLE 8--Influence coefficients for inside axial surface crack: $a / t=0.3$.

\begin{tabular}{|c|c|c|c|c|c|c|c|c|c|}
\hline $\begin{array}{l}\text { Aspect } \\
\text { Ratio }\end{array}$ & $\begin{array}{l}\text { Elliptic } \\
\text { Angle }\end{array}$ & $\begin{array}{l}\text { Ko } \\
\text { Uniform }\end{array}$ & $\begin{array}{l}\text { Kl } \\
\text { Linear }\end{array}$ & $\begin{array}{l}\text { K2 } \\
\text { Quadratic }\end{array}$ & $\begin{array}{l}\text { K3 } \\
\text { Cubic }\end{array}$ & $\begin{array}{l}\mathrm{K} 0 \\
t_{\mathrm{cl}}=6.35\end{array}$ & $\begin{array}{l}\mathrm{K} 1 \\
\mathrm{t}_{\mathrm{cl}}=6.35\end{array}$ & $\begin{array}{l}\mathrm{K} 0 \\
\mathrm{t}_{\mathrm{cl}}=3.96\end{array}$ & $\begin{array}{l}K 1 \\
t_{c l}=3.96\end{array}$ \\
\hline \multirow[t]{9}{*}{$2: 1$} & 0.00 & 0.723 & 0.127 & 0.048 & 0.026 & 0.404 & 0.188 & 0.334 & 0.176 \\
\hline & 17.4 & 0.708 & 0.203 & 0.083 & 0.042 & 0.102 & 0.049 & 0.056 & 0.025 \\
\hline & 29.1 & 0.690 & 0.264 & 0.126 & 0.068 & 0.058 & 0.028 & 0.034 & 0.016 \\
\hline & 40.9 & 0.680 & 0.326 & 0.185 & 0.114 & 0.043 & 0.021 & 0.026 & 0.013 \\
\hline & 52.6 & 0.673 & 0.381 & 0.251 & 0.177 & 0.036 & 0.018 & 0.022 & 0.011 \\
\hline & 64.4 & 0.668 & 0.423 & 0.310 & 0.242 & 0.032 & 0.016 & 0.020 & 0.010 \\
\hline & 76.1 & 0.665 & 0.452 & 0.355 & 0.297 & 0.030 & 0.015 & 0.018 & 0.009 \\
\hline & 87.9 & 0.662 & 0.478 & 0.385 & 0.331 & 0.028 & 0.014 & 0.017 & 0.009 \\
\hline & 90.0 & 0.662 & 0.482 & 0.391 & 0.337 & 0.027 & 0.014 & 0.017 & 0.009 \\
\hline \multirow[t]{9}{*}{$6: 1$} & 0.00 & 0.665 & 0.112 & 0.041 & 0.022 & 0.380 & 0.181 & 0.315 & 0.167 \\
\hline & 17.4 & 0.715 & 0.190 & 0.068 & 0.027 & 0.117 & 0.054 & 0.069 & 0.032 \\
\hline & 29.1 & 0.804 & 0.277 & 0.118 & 0.051 & 0.093 & 0.045 & 0.057 & 0.028 \\
\hline & 40.9 & 0.886 & 0.376 & 0.194 & 0.104 & 0.085 & 0.042 & 0.053 & 0.026 \\
\hline & 52.6 & 0.951 & 0.470 & 0.284 & 0.182 & 0.081 & 0.040 & 0.050 & 0.025 \\
\hline & 64.4 & 0.998 & 0.549 & 0.372 & 0.270 & 0.078 & 0.039 & 0.049 & 0.024 \\
\hline & 76.1 & 1.028 & 0.605 & 0.439 & 0.345 & 0.077 & 0.038 & 0.048 & 0.024 \\
\hline & 87.9 & 1.053 & 0.635 & 0.475 & 0.384 & 0.075 & 0.038 & 0.047 & 0.024 \\
\hline & 90.0 & 1.058 & 0.640 & 0.481 & 0.391 & 0.075 & 0.037 & 0.047 & 0.023 \\
\hline \multirow[t]{9}{*}{$10: 1$} & 0.00 & 0.562 & 0.085 & 0.029 & 0.014 & 0.344 & 0.168 & 0.290 & 0.153 \\
\hline & 17.4 & 0.707 & 0.176 & 0.052 & 0.016 & 0.128 & 0.059 & 0.078 & 0.037 \\
\hline & 29.1 & 0.848 & 0.276 & 0.104 & 0.016 & 0.110 & 0.054 & 0.068 & 0.034 \\
\hline & 40.9 & 0.962 & 0.389 & 0.188 & 0.082 & 0.102 & 0.051 & 0.064 & 0.032 \\
\hline & 52.6 & 1.051 & 0.498 & 0.288 & 0.169 & 0.098 & 0.049 & 0.062 & 0.031 \\
\hline & 64.4 & 1.115 & 0.590 & 0.385 & 0.265 & 0.096 & 0.048 & 0.060 & 0.030 \\
\hline & 76.1 & 1.157 & 0.653 & 0.460 & 0.346 & 0.095 & 0.047 & 0.060 & 0.030 \\
\hline & 87.9 & 1.183 & 0.685 & 0.496 & 0.387 & 0.094 & 0.047 & 0.059 & 0.029 \\
\hline & 90.0 & 1.187 & 0.691 & 0.503 & 0.394 & 0.094 & 0.047 & 0.059 & 0.029 \\
\hline
\end{tabular}


TABLE 9--Influence coefficients for inside axial surface crack: $\mathrm{a} / \mathrm{t}=0.5$.

\begin{tabular}{|c|c|c|c|c|c|c|c|c|c|}
\hline $\begin{array}{l}\text { Aspect } \\
\text { Ratio }\end{array}$ & $\begin{array}{l}\text { Elliptic } \\
\text { Angle }\end{array}$ & $\begin{array}{l}\text { K0 } \\
\text { Uniform }\end{array}$ & $\begin{array}{l}\mathrm{K} 1 \\
\text { Linear }\end{array}$ & $\begin{array}{l}\text { K2 } \\
\text { Quadratic }\end{array}$ & $\begin{array}{l}\text { K3 } \\
\text { Cubic }\end{array}$ & $\begin{array}{l}\mathrm{K} 0 \\
\mathrm{t}_{\mathrm{cl}}=6.35\end{array}$ & $\begin{array}{l}\mathrm{K} 1 \\
\mathrm{t}_{\mathrm{cl}}=6.35\end{array}$ & $\begin{array}{l}\mathrm{K} 0 \\
t_{\mathrm{cl}}=3.96\end{array}$ & $\begin{array}{l}\mathrm{K} 1 \\
t_{\mathrm{cl}}=3.96\end{array}$ \\
\hline \multirow[t]{9}{*}{$2: 1$} & 0.00 & 0.736 & 0.132 & 0.053 & 0.029 & 0.327 & 0.162 & 0.272 & 0.150 \\
\hline & 15.4 & 0.746 & 0.203 & 0.083 & 0.043 & 0.079 & 0.037 & 0.045 & 0.020 \\
\hline & 27.5 & 0.719 & 0.263 & 0.124 & 0.067 & 0.042 & 0.020 & 0.025 & 0.012 \\
\hline & 39.6 & 0.704 & 0.327 & 0.183 & 0.112 & 0.029 & 0.014 & 0.018 & 0.009 \\
\hline & 51.7 & 0.693 & 0.383 & 0.249 & 0.175 & 0.023 & 0.012 & 0.015 & 0.007 \\
\hline & 63.7 & 0.685 & 0.426 & 0.311 & 0.242 & 0.021 & 0.010 & 0.013 & 0.006 \\
\hline & 75.8 & 0.681 & 0.456 & 0.357 & 0.299 & 0.019 & 0.009 & 0.012 & 0.006 \\
\hline & 87.9 & 0.676 & 0.483 & 0.389 & 0.334 & 0.018 & 0.009 & 0.011 & 0.006 \\
\hline & 90.0 & 0.676 & 0.488 & 0.395 & 0.340 & 0.017 & 0.009 & 0.011 & 0.005 \\
\hline \multirow[t]{9}{*}{$6: 1$} & 0.00 & 0.758 & 0.142 & 0.059 & 0.033 & 0.322 & 0.163 & 0.268 & 0.149 \\
\hline & 15.4 & 0.814 & 0.213 & 0.083 & 0.040 & 0.091 & 0.041 & 0.054 & 0.025 \\
\hline & 27.5 & 0.908 & 0.302 & 0.132 & 0.065 & 0.070 & 0.034 & 0.043 & 0.021 \\
\hline & 39.6 & 0.998 & 0.405 & 0.208 & 0.116 & 0.065 & 0.032 & 0.040 & 0.020 \\
\hline & 51.7 & 1.069 & 0.504 & 0.300 & 0.195 & 0.062 & 0.031 & 0.039 & 0.019 \\
\hline & 63.7 & 1.120 & 0.588 & 0.392 & 0.285 & 0.061 & 0.030 & 0.038 & 0.019 \\
\hline & 75.8 & 1.153 & 0.647 & 0.463 & 0.363 & 0.060 & 0.030 & 0.038 & 0.019 \\
\hline & 87.9 & 1.182 & 0.679 & 0.500 & 0.404 & 0.059 & 0.029 & 0.037 & 0.018 \\
\hline & 90.0 & 1.187 & 0.685 & 0.506 & 0.411 & 0.059 & 0.029 & 0.037 & 0.018 \\
\hline \multirow[t]{9}{*}{$10: 1$} & 0.00 & 0.666 & 0.119 & 0.049 & 0.028 & 0.302 & 0.156 & 0.254 & 0.140 \\
\hline & 15.4 & 0.822 & 0.208 & 0.077 & 0.033 & 0.097 & 0.044 & 0.060 & 0.028 \\
\hline & 27.5 & 0.995 & 0.316 & 0.131 & 0.056 & 0.086 & 0.042 & 0.054 & 0.027 \\
\hline & 39.6 & 1.138 & 0.440 & 0.216 & 0.112 & 0.083 & 0.041 & 0.052 & 0.026 \\
\hline & 51.7 & 1.251 & 0.560 & 0.321 & 0.198 & 0.083 & 0.041 & 0.052 & 0.026 \\
\hline & 63.7 & 1.335 & 0.662 & 0.425 & 0.298 & 0.083 & 0.041 & 0.052 & 0.026 \\
\hline & 75.8 & 1.390 & 0.734 & 0.506 & 0.383 & 0.083 & 0.041 & 0.052 & 0.026 \\
\hline & 87.9 & 1.423 & 0.770 & 0.546 & 0.427 & 0.083 & 0.041 & 0.052 & 0.026 \\
\hline & 90.0 & 1.429 & 0.776 & 0.553 & 0.434 & 0.083 & 0.041 & 0.052 & 0.026 \\
\hline
\end{tabular}

TABLE 10--Influence coefficients for inside circumferential surface crack: $\mathrm{a} / \mathrm{t}=0.5$.

\begin{tabular}{llllllllll}
\hline $\begin{array}{l}\text { Aspect } \\
\text { Ratio }\end{array}$ & Elliptic & K0 & K1 & K2 & $\mathrm{K} 3$ & $\mathrm{~K} 0$ & $\mathrm{~K} 1$ & $\mathrm{~K} 0$ & $\mathrm{~K} 1$ \\
\hline $2: 1$ & 0.00 & 0.741 & 0.134 & 0.054 & 0.030 & 0.324 & 0.162 & 0.269 & 0.151 \\
& 15.4 & 0.750 & 0.205 & 0.084 & 0.044 & 0.079 & 0.038 & 0.045 & 0.020 \\
& 27.5 & 0.721 & 0.264 & 0.124 & 0.067 & 0.042 & 0.020 & 0.025 & 0.012 \\
& 39.6 & 0.706 & 0.328 & 0.183 & 0.112 & 0.029 & 0.014 & 0.018 & 0.009 \\
& 51.7 & 0.698 & 0.384 & 0.250 & 0.175 & 0.024 & 0.012 & 0.015 & 0.007 \\
& 63.7 & 0.692 & 0.430 & 0.312 & 0.243 & 0.021 & 0.010 & 0.013 & 0.007 \\
& 75.8 & 0.686 & 0.461 & 0.360 & 0.301 & 0.019 & 0.010 & 0.012 & 0.006 \\
& 87.9 & 0.682 & 0.488 & 0.392 & 0.336 & 0.020 & 0.010 & 0.012 & 0.006 \\
& 90.0 & 0.682 & 0.493 & 0.398 & 0.343 & 0.020 & 0.009 & 0.013 & 0.006 \\
\hline $6: 1$ & 0.00 & 0.727 & 0.132 & 0.053 & 0.030 & 0.315 & 0.161 & 0.262 & 0.147 \\
& 15.4 & 0.786 & 0.205 & 0.079 & 0.037 & 0.087 & 0.039 & 0.052 & 0.024 \\
& 27.5 & 0.882 & 0.295 & 0.128 & 0.062 & 0.067 & 0.032 & 0.041 & 0.020 \\
& 39.6 & 0.974 & 0.398 & 0.205 & 0.114 & 0.062 & 0.031 & 0.038 & 0.019 \\
& 51.7 & 1.049 & 0.499 & 0.298 & 0.193 & 0.060 & 0.030 & 0.037 & 0.019 \\
& 63.7 & 1.103 & 0.584 & 0.390 & 0.284 & 0.058 & 0.029 & 0.036 & 0.018 \\
& 75.8 & 1.138 & 0.644 & 0.462 & 0.362 & 0.057 & 0.029 & 0.036 & 0.018 \\
& 87.9 & 1.166 & 0.676 & 0.499 & 0.403 & 0.058 & 0.029 & 0.036 & 0.018 \\
& 90.0 & 1.171 & 0.682 & 0.506 & 0.410 & 0.058 & 0.029 & 0.036 & 0.018 \\
\hline $10: 1$ & 0.00 & 0.616 & 0.101 & 0.040 & 0.023 & 0.291 & 0.152 & 0.247 & 0.138 \\
& 15.4 & 0.770 & 0.195 & 0.071 & 0.028 & 0.090 & 0.039 & 0.055 & 0.026 \\
& 27.5 & 0.936 & 0.301 & 0.125 & 0.053 & 0.078 & 0.038 & 0.049 & 0.024 \\
& 39.6 & 1.076 & 0.424 & 0.211 & 0.109 & 0.075 & 0.037 & 0.047 & 0.024 \\
& 51.7 & 1.190 & 0.544 & 0.315 & 0.196 & 0.075 & 0.037 & 0.047 & 0.023 \\
& 63.7 & 1.275 & 0.647 & 0.420 & 0.295 & 0.075 & 0.037 & 0.047 & 0.023 \\
& 75.8 & 1.330 & 0.719 & 0.501 & 0.381 & 0.075 & 0.037 & 0.047 & 0.023 \\
& 87.9 & 1.363 & 0.755 & 0.542 & 0.425 & 0.075 & 0.037 & 0.047 & 0.024 \\
& 90.0 & 1.368 & 0.762 & 0.549 & 0.433 & 0.075 & 0.037 & 0.047 & 0.024 \\
\hline
\end{tabular}




\section{COMPARISON WITH OTHER INVESTIGATORS}

A comparison of some of the 3-D influence coefficients generated in this study with similar coefficients obtained by other investigators [3,4] is shown in Figs. 7-9. To compare the coefficients with those obtained from Raju and Newman [3], the influence coefficients in Figs. 7 and 8 represent $K_{j}^{*}$ values multiplied by $\sqrt{Q}$, where the shape factor (Q) is the square of the complete elliptic integral of the second kind. The shape factor for an elliptical crack is approximated by the following equation:

$$
\mathrm{Q}=1+1.464(\mathrm{a} / \mathrm{c})^{1.65} .
$$

As indicated in Figs. 7 and 8, the coefficients being compared pertain to axially oriented, inner-surface flaws in a long cylinder with $a / t=0.2$ and 0.5 and aspect ratios of $2: 1$ and $10: 1$.
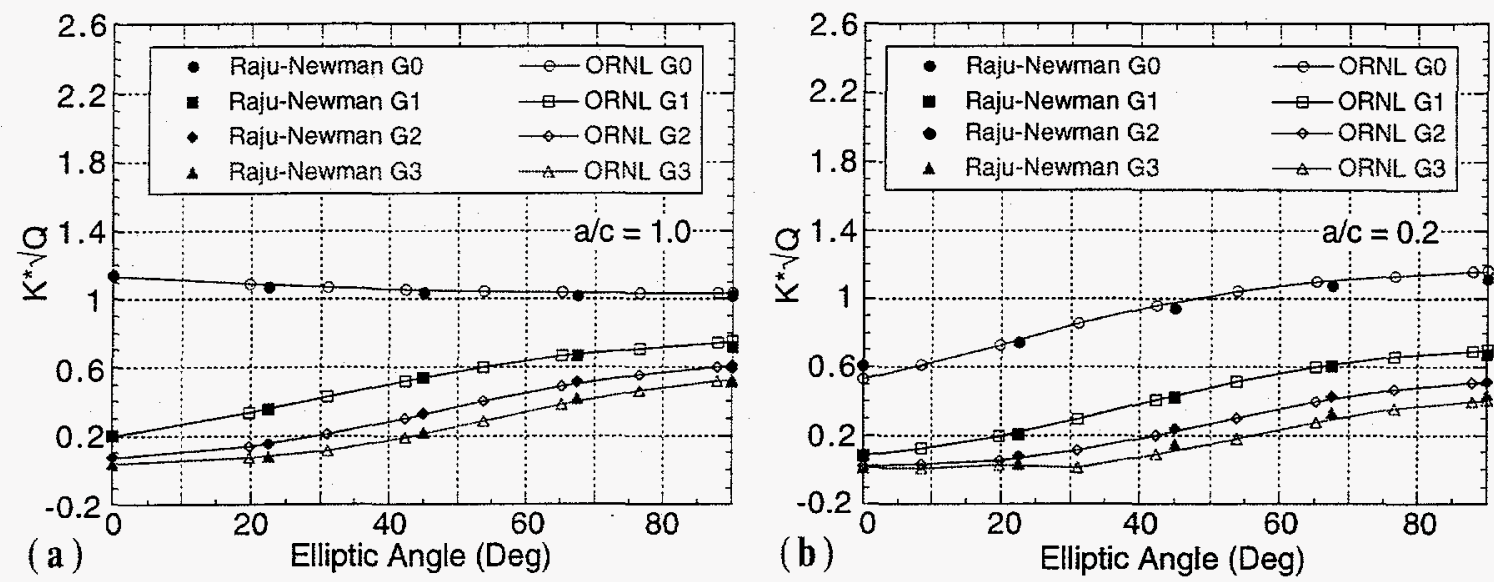

FIG. 7--Influence coefficients for axially oriented semielliptical flaws $(a / t=0.2)$ on inner surface of cylinder: (a) 2:1 aspect ratio; (b) 10:1 aspect ratio
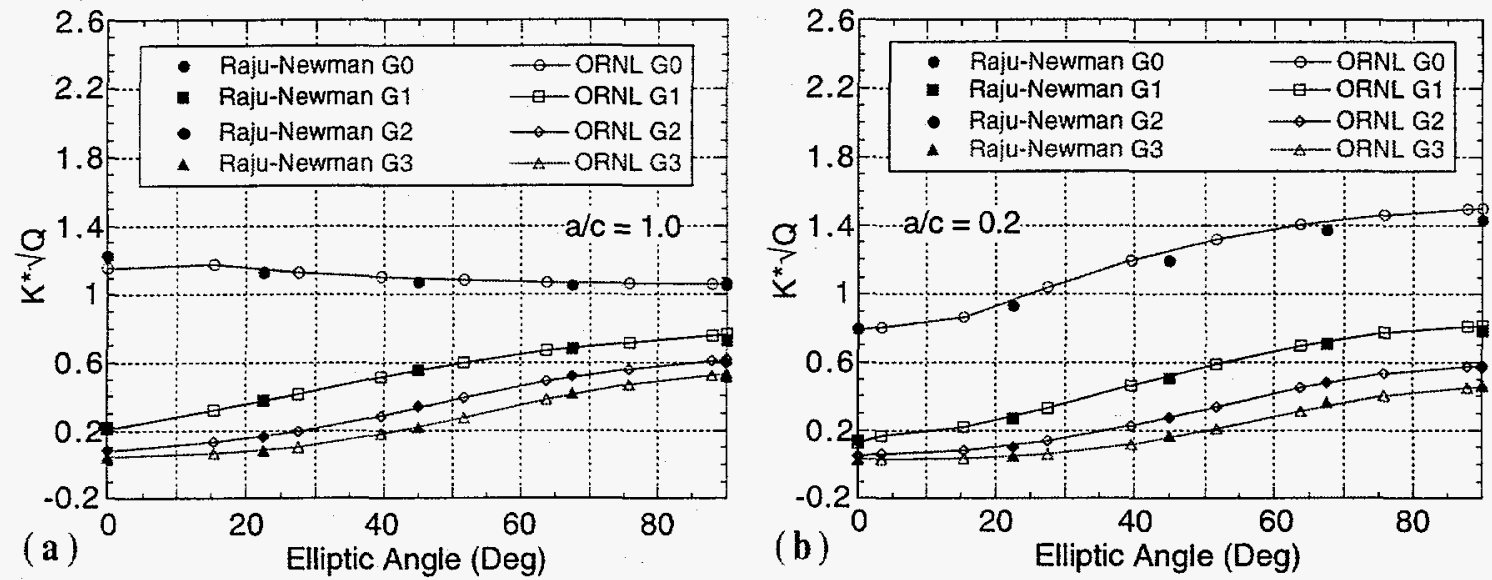

FIG. 8--Influence coefficients for axially oriented semielliptical flaws $(\mathrm{a} / \mathrm{t}=0.5)$ on inner surface of cylinder: (a) 2:1 aspect ratio; (b) 10:1 aspect ratio. 
The coefficients in Fig. 9 (Ref. 4) are $K_{j}^{*}$ values for an aspect ratio of $6: 1$ and $a / t=0.5$. The results of all the comparisons indicate good agreement. The other investigators $[3,4]$ did not publish SIFICs for the shallower flaw depths so a comparison could not be made for $\mathrm{a} / \mathrm{t}$ ratios less than 0.2 .

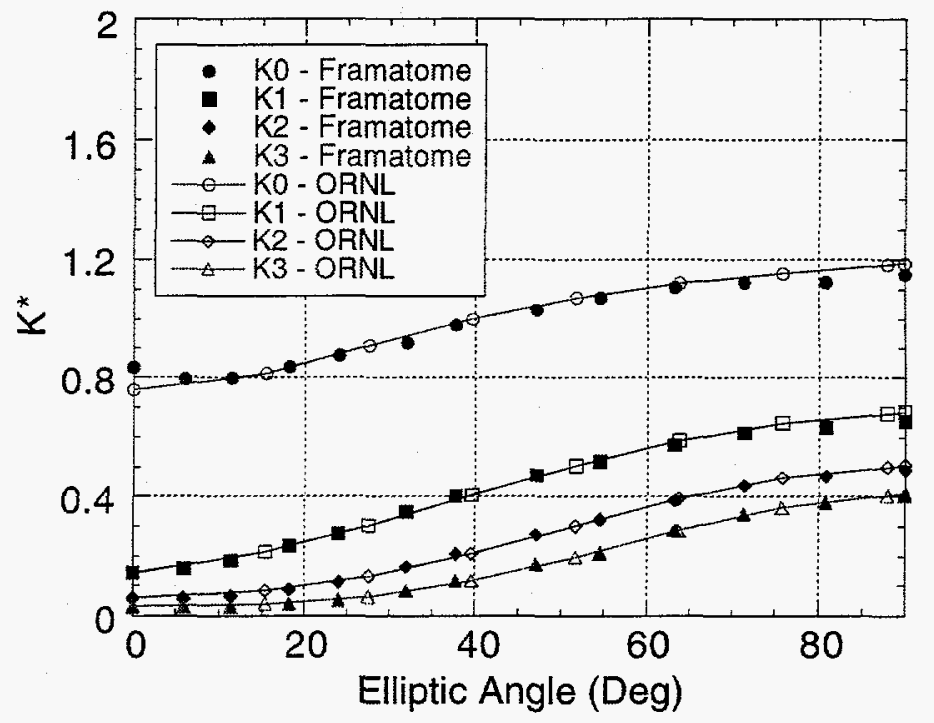

FIG. 9--Influence coefficients for an axially oriented semielliptical flaw with $a / t=0.5$ and $6: 1$ aspect ratio.

\section{SUMMARY AND CONCLUSIONS}

The results of the present study show that there are essentially no differences in SIFICs for finite inner-surface axial and finite inner-surface circumferential flaws having flaw depths $(0.01 \leq a / t \leq 0.5)$ and flaw aspect ratios $(2 \mathrm{c} / \mathrm{a}=2,6,10)$. This important finding implies that SIFICs for axial flaws can be used for circumferential flaws as deep as halfway through the vessel wall with very little error provided that $2 \mathrm{c} / \mathrm{a} \leq 10$. As might be expected, the greatest difference $(\sim 5 \%)$ between the two is obtained for flaw geometries with an $a / t=0.5$ and $2 \mathrm{c} / \mathrm{a}=10$. For implementation into fracture mechanics codes, it is recommended that SIFICs calculated for axial flaws be used for circumferential flaws having an $a / t<0.5$ and $2 c / a \leq 10$. The SIFICs given in Table 10 should be utilized for circumferential flaws having an $\mathrm{a} / \mathrm{t}=0.5$.

\section{ACKNOWLEDGMENTS}

Research sponsored by the Office of Nuclear Regulatory Research, U.S. Nuclear Regulatory Commission under Interagency Agreement 1886-8011-9B with the U.S. Department of Energy under Contract DE-AC05-84OR21400 with Martin Marietta Energy Systems, Inc.

The submitted manuscript has been authored by a contractor of the U.S. Government No. DE-AC05-84OR21400. Accordingly, the U.S. Government retains a nonexclusive, royalty-free license to publish or reproduce the published form of this contribution, or allow others to do so, for U.S. Government purposes. 


\section{REFERENCES}

[1] DeLorenzi, H. G., "3-D Elastic-Plastic Fracture Analysis with ADINA," Computers and Structures, Vol. 13, 1981, pp. 613-621.

[2] Bass, B. R. and Bryson, J. W., "Applications of Energy Release Rate Techniques to Part-Through Cracks in Experimental Pressure Vessels," J. Pressure Vessel Tech., Vol. 104, November 1982, pp. 308-316.

[3] Raju, I. S. and Newman, J. C., Jr., "Stress-Intensity Factor Influence Coefficients For Internal and External Surface Cracks in Cylindrical Vessels," Aspects of Fracture Mechanics in Pressure Vessels and Piping, ASME Pub. PVP, Vol. 58, American Society of Mechanical Engineers, 1982, pp. 37-48.

[4] Lance,C., Robisson, F., and Pellissier-Tanon, A., "Influence of Cladding on the Values of $\mathrm{K}_{\mathrm{I}}$ for Emerging Defects in the Vessel Beltline," Framatome (1981), ORNL-TR4960, Oak Ridge National Laboratory, Oak Ridge, TN, February 1983.

[5] Ball, D. G., Bass, B. R., Bryson, J. W., Cheverton, R. D., and Drake, J. B., "Stress-Intensity-Factor Influence Coefficients for Surface Flaws in Pressure Vessels," USNRC Report NUREG/CR-3723 (ORNL/CSD/TM-216), U. S. Nuclear Regulatory Commission, Washington DC, February 1985.

[6] Dickson, T. L., "FAVOR: A New Fracture Mechanics Code for Reactor Pressure Vessels Subjected to Pressurized Thermal Shock," Pressure Vessel Integrity, ASME Pub. PVP, Vol. 250, American Society of Mechanical Engineers, 1993, pp. 3-10.

[7] Bueckner, H. F., "A Novel Principle for the Computation of Stress Intensity Factors," Z. angew. Math. Mech.,Vol. 50, 1970, pp. 529-546.

[8] ABAQUS Theory Manual, Version 5.2, Hibbit, Karlson and Sorensen, Inc., Providence, RI, 1992.

[9] Bass, B. R. and Bryson, J. W., "Applications of Energy Release Rate Techniques to Part-Through Cracks in Plates and Cylinders, Volume 1, ORMGEN-3D: A Finite Element Mesh Generator for 3-Dimensional Crack Geometries," USNRC Report NUREG/CR2997/V1 (ORNL/TM-8527), December 1982.

[10] Timoshenko, S., Theory of Plates and Shells, McGraw-Hill, New York, 1940.

[11] Bishop, B. A., Dickson, T. L., and Simonen, F. A., "Benchmarking of Probabilistic Fracture Mechanics Analyses of Reactor Vessels Subjected to Pressurized Thermal Shock (PTS) Loading," Electric Power Research Institute, Research Project 2975-5, Final Report, July 1993.

[12] Cheverton, R. D., "Thermal Shock Studies Associated with Injection of Emergency Core Coolant Following a Loss-of-Coolant Accident in PWRs, " Nuclear Safety, Vol. 19, No. 1, January-February 1978.

[13] Bryson, J. W. and Dickson, T. L., "Stress-Intensity-Factor Influence Coefficients for Axial and Circumferential Flaws in Reactor Pressure Vessels, "Pressure Vessel Integrity, ASME Pub. PVP-, Vol. 250, American Society of Mechanical Engineers, 1993, pp. $77-87$. 